\title{
Common Peroneal Nerve Grafting to Repair the Tibial Nerve as a Salvage Procedure in the Treatment of Sciatic Nerve Injury with Long-Segment Defects
}

\author{
Haodong Lin, MD, $\mathrm{PhD}^{1}$ Desong Chen, $\mathrm{MD}^{2} \quad$ Chunlin Hou, $\mathrm{MD}^{1}$ \\ ${ }^{1}$ Department of Orthopedic Surgery, Changzheng Hospital, \\ The Second Military Medical University, Shanghai, China \\ 2 Department of Hand Surgery, Ningbo No. 6 Hospital, \\ Zhejiang, China

\begin{abstract}
Address for correspondence Chunlin Hou, MD, Department of Orthopedic Surgery, Changzheng Hospital, The Second Military Medical University, Fengyang Road 415, Shanghai 200003, China (e-mail: chunlin_hou@163.com).
\end{abstract}

J Reconstr Microsurg Open 2018;3:e41-e45.

\begin{abstract}
Background In sciatic nerve neuropathies, when direct nerve repair is impossible due to a large gap, nerve grafting can be performed. However, the diameters of traditional autologous nerve grafts are too small to cover the whole cross-sectional area of the sciatic nerve. The aim of this study is to present the outcome of common peroneal nerve grafting to repair the tibial nerve in eight patients with sciatic nerve injuries, showing long defects of more than $10 \mathrm{~cm}$.

Methods Between 2007 and 2013, the common peroneal nerve was used as an autograft to repair the tibial nerve in eight patients with complete high sciatic nerve injury with long defects. There were 6 men and 2 women with an average age of 31 years (range: $17-44$ years). Muscle strength was evaluated using the British Medical Research Council scale. The Semmes-Weinstein monofilament test was used for sensory evaluation.

Results The follow-up time for patients ranged from 36 to 60 months, with an average of 48.75 months. Tibial nerve motor function was "good" or "very good" (M3-M4) in five out of eight patients (55.6\%). Plantar flexion was not adequate in the rest of the patients. Sensory recovery was "good" or "very good" (S2-S3) in six patients and

Keywords

- sciatic nerve

- nerve graft

- plantar flexion

"inadequate" (S4) in two patients.

Conclusion In cases where there were extensive gaps in the sciatic nerve, using the common peroneal nerve as an autograft to repair the tibial nerve provides an alternative to traditional nerve graft repair.
\end{abstract}

Lower extremity nerve injuries are relatively less common than nerve injuries to the upper extremities. ${ }^{1-3}$ Sciatic nerve lesions comprise the largest subset of lower extremity nerve injuries. In sciatic nerve neuropathies, when direct nerve repair is impossible due to a large gap, nerve grafting can be performed. For a donor nerve, the sural nerve is considered ideal for grafting. The maximum piece of length of nerve one can obtain is $30 \mathrm{~cm}$, whereas multiple strands of the nerve are required to cover the whole cross-sectional area of the sciatic nerve. When the defect of injured sciatic nerve is more than $10 \mathrm{~cm}$, only 4 to 6 fascicular grafts (numbers of grafts) can be obtained even if the sural nerve is taken from both legs, because its length is approximately $30 \mathrm{~cm}$. In this situation, only partial coverage of the sciatic nerve is possible, thus needing more donor nerve. received

December 28, 2017 accepted after revision April 7, 2018
DOI https://doi.org/

10.1055/s-0038-1653951. ISSN 2377-0813.
Copyright $\odot 2018$ by Thieme Medical Publishers, Inc., 333 Seventh Avenue, New York, NY 10001, USA. Tel: +1(212) 584-4662.
License terms

(요 (1) $\Theta \circledast$ 
After similar severe injury to the sciatic nerve or its division into the peroneal and tibial nerves, recovery in the peroneal nerve is inferior to that of the tibial nerve. ${ }^{4-7}$ The functional recovery, especially of the peroneal nerve (muscle grade more than 3 ), is approximately $14 \%$ for grafts and the value of the attempted repair of the peroneal nerve has been questioned. ${ }^{4,5,7}$ For tibial nerve reconstruction, the percentage has been reported to be higher, as $67 \%$ for grafts. ${ }^{6}$ The tibial nerve is a major branch of the sciatic nerve, and it contributes to the motor function of the posterior leg and to the intrinsic muscles of the foot. Important sensory supply to the foot comes through the tibial nerve. Thus, injury of the tibial nerve will result in a lack of foot or plantar flexion, a loss of toe flexion and inversion, and a lack of sensation on the sole of the foot. An insensitive foot will be problematic until enough sensation returns. Therefore, tibial nerve repair at any level is always advisable.

With the abovementioned reasons, in this article, the common peroneal nerve was sacrificed as autograft to repair the tibial nerve in patients with a large gap of the sciatic nerve. This surgical method aimed to restore some important functions within the lower extremities and provide an alternative approach for treating long segment defects in the sciatic nerve.

\section{Methods}

\section{Patients}

Eight patients with complete high sciatic nerve injury were treated with a nerve autograft during a 6-year interval from 2007 to 2013. All patients showed complete paralysis of the sciatic nerve, with an injury located at the thigh level, free from vascular trauma or soft-tissue defects. The patients for the study (-Table 1) comprised six men and two women with an average age of 31 years (range: 17-44 years). The cause of the injury was traffic accidents in four patients, tumor excision in three patients, and gunshot wound injury in one patient. The average delay from the injury to surgery was 6 months (range: 0.5-12 months). Ethics approval was granted by our institution and informed consent was obtained from all patients prior to study commencement.

Preoperative and postoperative evaluation included motor and sensory examinations of the lower extremity and electromyography. The clinical examination included tests of the lower extremity musculature (specifically the tibial nerve foot flexors, gastrocnemius, and soleus) which are innervated by the tibial and peroneal divisions of the sciatic nerve, and were always performed in comparison with the contralateral normal side. Muscle strength was evaluated using the British Medical Research Council (MRC) scale. The Semmes-Weinstein monofilament test was used for sensory evaluation. Motor and sensitivity improvements were graded on a five-point scale, ranked accordingly: M5 and S1 were considered as excellent; M4 and S2 as very good; M3 and S3 as good, but represented an improvement which was not adequate for normal function; and M0-M2 and S4-S5 were classified as inadequate. ${ }^{8,9}$

The evaluation of patients in the postoperative period was based on serial clinical examination and electrodiagnostic studies. Each patient was followed up after 6 weeks, after 3 months, and thereafter at 3-month intervals up to 24 months, after which they returned at 6-month intervals.

\section{Surgical Approach}

Operations were performed under general anesthesia using an operating microscope (magnification $\times 10$ ). With the patient placed prone, an undulating incision was made in the posterior midline thigh along the medial border of the lateral hamstring tendon and was extended curvilinearly as needed, either into the lateral buttock crease or toward the popliteal fossa. The long head of the biceps were angled across the upper thigh and retracted superiorly or inferiorly to expose the proximal thigh-level sciatic nerve. Exposure of the inferior border of the gluteus maximus muscle was helpful in visualizing the sciatic nerve at the buttock crease. The gluteus could be undermined and retracted superiorly using a medium-sized Richardson retractor, thus preserving the branches to the lateral short head of the biceps femoris that arise from the peroneal division distal to the buttock crease.

Fine dissecting microscissors or a scalpel blade were used for sharp dissection of the sciatic nerve. The presumed lesion site was approached in a circumferential manner, alternating from the superiorly more normal proximal nerve toward the lesion and distally from the degenerated intact nerve, likewise toward the lesion. Regions of bleeding at the epineurial

Table 1 Patient characteristics and functional recovery

\begin{tabular}{|c|c|c|c|c|c|}
\hline Patient no. & Etiology & Length of gap (cm) & Follow-up (mo) & Tibial nerve motor function & Plantar sensory \\
\hline 1 & Traffic accident & 11.5 & 42 & M4 & $\mathrm{S} 2$ \\
\hline 2 & Tumor excision & 10.4 & 48 & $\mathrm{M} 2$ & S3 \\
\hline 3 & Traffic accident & 12.4 & 48 & M4 & $\mathrm{S} 2$ \\
\hline 4 & Traffic accident & 13.6 & 60 & M2 & S4 \\
\hline 5 & Tumor excision & 10.8 & 54 & M3 & S3 \\
\hline 6 & Gunshot wound & 11.2 & 36 & M1 & S4 \\
\hline 7 & Tumor excision & 12.7 & 54 & M4 & S2 \\
\hline 8 & Traffic accident & 11.4 & 48 & M3 & S3 \\
\hline
\end{tabular}


site were coagulated with irrigating bipolar forceps, requiring enhanced magnification through a surgical loupe or microscope. A clear surgical exposure of the viable nerve both proximal and distal to the lesion was prepared, and proximal and distal nerve stumps or nontransmitting nerve segments were resected to healthy epineurium with normal fascicular and vascular architecture (-Fig. 1A). The final defect size was determined while the knee was in full extension, when preparing for the nerve grafting procedure to repair the nerve defects, which were more than $10 \mathrm{~cm}$ in all patients.
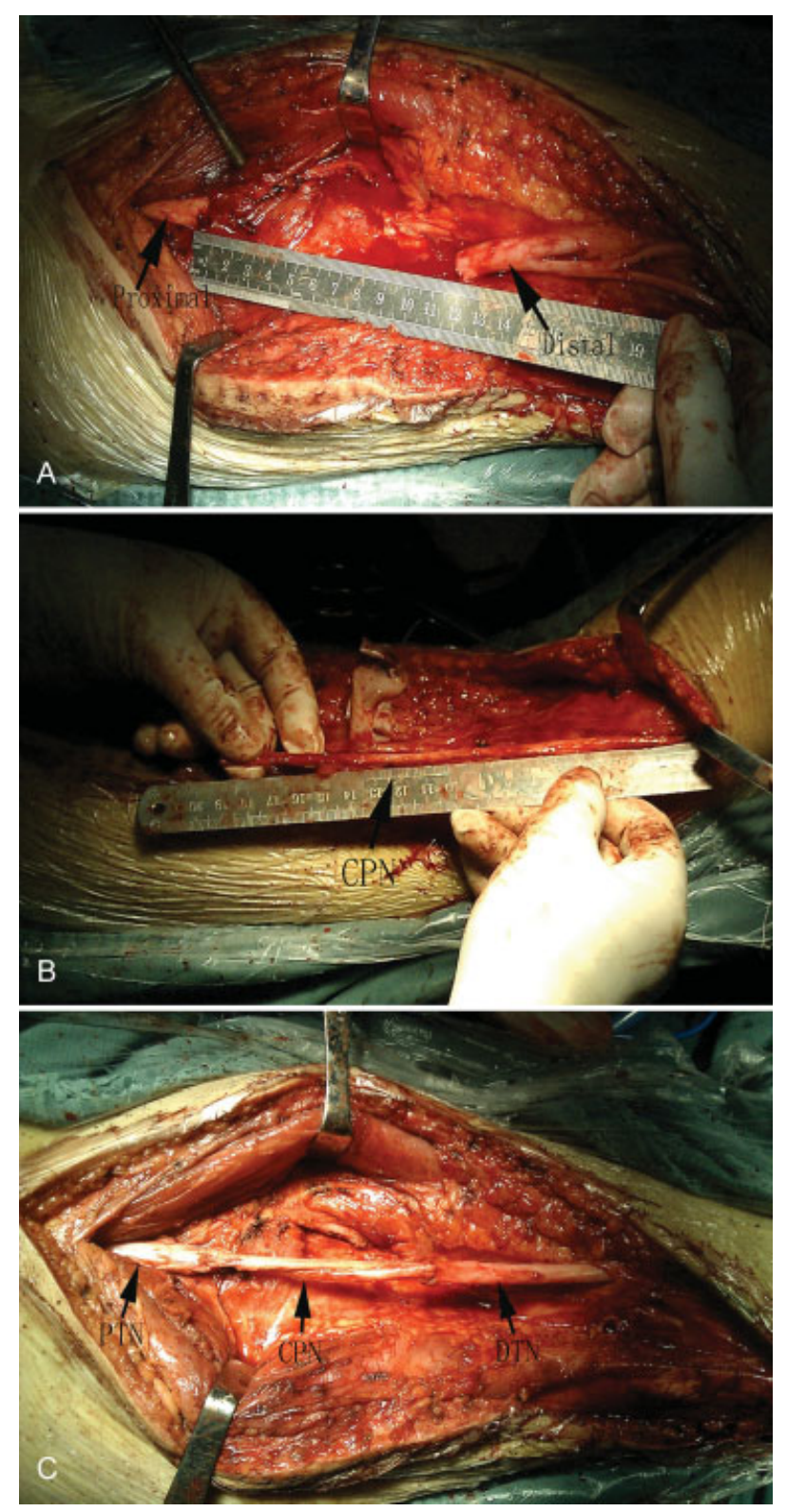

Fig. 1 Representative intraoperative photographs of common peroneal nerve grafting to repair the tibial nerve in a patient with a longsegment sciatic nerve injury. (A) After trimming the nerve back to healthy nerve endings so that it is free of any surrounding scar tissue proximally and distally, the nerve defect is $11.5 \mathrm{~cm}$. (B) A nerve graft is taken from the common peroneal nerve (CPN). (C) The proximal end of the tibial nerve (PTN) and distal end of the tibial nerve (DTN) are bridged by the common peroneal nerve.
Two interfascicular nerve autografts, harvested from the distal part of the common peroneal nerve ( $\mathbf{- F i g . ~ 1 B ) , ~ w e r e ~}$ placed between the prepared proximal and distal stumps of the tibial nerve. The common peroneal nerve was used as an autograft to repair the tibial nerve in all cases, using the injured leg as the source. Two grafts were performed, such that the entire cross-sectional area of the sciatic nerve was covered with the interfascicular nerve autograft (-Fig. 1C). The autografts were sutured to these areas under microscopic magnification using 9-0 nylon. The wound was closed with three layers of sutures, and an external drain was inserted. After surgery, broad-spectrum antibiotics were given for 3 days.

Patients received routine postoperative care. In most cases, patients were discharged from the hospital approximately 1 week after surgery and then moved to a rehabilitation clinic 2 weeks postoperatively. Physical therapy began 3 weeks postoperatively. To obtain the optimal functional result after nerve transfer, the rehabilitation program included motor reeducation to initiate recruitment of the weak reinnervated soleus and gastrocnemius muscle and to establish new motor pattern.

\section{Results}

\section{Clinical Data}

There was no complication in the early postoperative period. Local wound infection was encountered in one patient and was treated with local wound care and systemic antibiotics. The graft material was 10.4 to $13.6 \mathrm{~cm}$ in length, with a mean length of $11.75 \pm 1.07 \mathrm{~cm}$. The follow-up period ranged between 36 and 60 months.

\section{Recovery after Nerve Repair}

Electrophysiological examination revealed "nascent" motor unit potentials in the soleus and gastrocnemius approximately 12 months after the operation in most patients. This indicated the successful regeneration of the tibial nerve into the soleus and gastrocnemius. Recovery of plantar flexion was first evident at 15 to 18 months after nerve repairs. Tibial nerve motor function (soleus and gastrocnemius muscle strength) was M4 (according to the British MRC system) in three patients and M3 in two patients. These patients did not require any bracing to stabilize the lower leg and were able to walk without support. The plantar flexion was not sufficient for the rest of the patients. The postoperative results of the electrodiagnostic examinations were concordant with the physical examinations, with sensory recovery graded at S2 (very good) in three patients, S3 (good) in three patients, and S4 (inadequate) in two patients.

\section{Discussion}

\section{Anatomical Basis for the Surgical Procedures}

Anatomically, the sciatic nerve is the longest and the largest diameter nerve of the body. It is composed of independent tibial and peroneal branches. ${ }^{10}$ Each of these sciatic nerve branches can easily be separately dissected for surgical 
procedures such as nerve repair and grafting. However, functional outcomes and recovery profiles after surgical interventions on the two branches differ, with the prognosis after treatment of the peroneal nerve not as favorable as that following treatment of the tibial division. ${ }^{7,11}$ The peroneal division of the sciatic nerve, which is usually composed of one major bundle and located laterally to the tibial division, is more susceptible to trauma. It has a poor blood supply and less protective connective tissue compared with the tibial nerve. Therefore, the tibial nerve is the preferred target for the repair of sciatic nerve injury. ${ }^{12-14}$

The tibial nerve provides sensation to the sole of the foot and branches of the gastrocnemius/soleus muscle mass, to the posterior tibialis muscle, and to the flexors of the toes. Injuries of the tibial nerve or tibial division result in a lack of foot flexion and sensation on the sole of the foot, as well as foot inversion and toe flexion. Blisters, ulcers, and even osteomyelitis can occur as a consequence of an insensitive foot. Therefore, the primary goal of sciatic nerve repair is the restoration of the protective sensibility of the sole. Secondary goals include restoration of the plantar flexion to achieve push-off during walking for the tibial division and correction of drop foot for the peroneal division. ${ }^{15}$

Autografting is the optimal reconstruction method for many nerve gaps, because the nerve architecture that is retained serves as a regenerative scaffold. Autologous nerve grafts are normally performed using donor sites such as the sural, superficial radial, and medial cutaneous sensory nerves. ${ }^{16}$ However, in sciatic nerve neuropathies, the diameters of these traditional autologous nerve grafts are too small to cover the whole cross-sectional area of the sciatic nerve, even with multiple strands of the nerve, and hence more donor nerve is needed. In the treatment of total brachial plexus avulsion of the upper limb, the cervical 7 nerve root is usually used to repair the median nerve or the radial nerve. ${ }^{17}$ In this procedure, the ulnar nerve is usually sacrificed to be used as a nerve graft because its integrity is not considered critical for a good functional outcome. Similarly, we reasoned in this study that when the sciatic nerve is injured, the peroneal nerve could be used as a source of nerve grafts to repair the tibial nerve.

\section{Assessment of Results}

The results show that tibial nerve motor function (soleus and gastrocnemius muscle strength) was recovered to a functional level in five out of eight patients (M4 in 3 patients and M3 in 2 patients), while sensory recovery was adequate in six patients (S2 in 3 patients and S3 in 3 patients). These patients did not require any bracing to stabilize the lower leg and were able to walk without support. These results indicate that the surgical procedure presented in this study is an effective treatment method for sciatic nerve injury with long defects. Although part of the lower limb function will be lost with this procedure, more important functions can be recovered instead.

\section{Advantages of the Surgical Procedures}

The surgical procedures described in this article are straightforward and can be performed without difficulty by a neurosurgeon. Although the diameter of the peroneal nerve is smaller than that of the tibial nerve, it is nonetheless much larger than that of traditional autologous nerve grafts, such as the sural nerve. We found that two bundles of peroneal nerves were large enough to cover the whole cross-sectional area of the tibial nerve. When autologous peroneal nerve bundles were used, more myelinated nerve fibers from the proximal end of the tibial nerve could run across the nerve graft to the distal end of the tibial nerve than when traditional autologous nerve grafts were used. Furthermore, the function of the tibial nerve recovered better than when traditional autologous nerve grafts were used.

\section{Factors Influencing Outcome}

Functional recovery after graft repair depends on the severity of injury and also on the length of the grafts. ${ }^{5,18}$ Prior to this study, there have been only a few reports in the literature regarding the relationship between graft length and functional outcomes, and little consensus on the critical length of the nerve graft affecting the final recovery rate. Kim and Kline found that a good functional recovery was not observed with a graft length of more than $12 \mathrm{~cm}$, and therefore argued that a nerve graft length of 5 to $12 \mathrm{~cm}$ is required for a good outcome. ${ }^{19}$ Lee et al reported good to excellent motor recovery in $83 \%$ of patients who had a large gap of the radial nerve (more than $9 \mathrm{~cm}$ ) when sural nerve autografts were sutured to the areas that have motor-dominant fascicles. ${ }^{20}$ Our patients had sciatic nerve defects ranging from 10.4 to $13.6 \mathrm{~cm}$ and show outcomes similar to the study of Lee et al. Therefore, we believe that even when the peripheral nerve defect is over $10 \mathrm{~cm}$, repairing the defect can still be of benefit to the patient.

\section{Conclusion}

The results of this study confirm that in patients with a large gap in their sciatic nerve, the common peroneal nerve can be sacrificed as an autograft to repair the tibial nerve, helping to restore some important functional activity to the lower extremities. It can also be used successfully to bridge nerve defects longer than $10 \mathrm{~cm}$, where conventional nerve grafts usually fail. For these reasons, we advocate this procedure as an alternative approach for the treatment of long-segment sciatic nerve defects.

\section{Funding}

This work was supported by the Shuguang Program of Shanghai Education Development Foundation and Shanghai Municipal Education Commission (grant number 15SG34) and the Project of Research doctor of Shanghai Changzheng Hospital (grant 201712).

\section{Conflict of Interest}

None.

\section{References}

1 Noble J, Munro CA, Prasad VS, Midha R. Analysis of upper and lower extremity peripheral nerve injuries in a population of patients with multiple injuries. J Trauma 1998;45(01):116-122 
2 Lin H, Xu Z, Liu Y, Chen A, Hou C. The effect of severing L6 nerve root of the sacral plexus on lower extremity function: an experimental study in rhesus monkeys. Neurosurgery 2012;70(01):170-177

3 Robinson LR. Traumatic injury to peripheral nerves. Muscle Nerve 2000;23(06):863-873

4 Roganovic Z, Pavlicevic G. Difference in recovery potential of peripheral nerves after graft repairs. Neurosurgery 2006;59(03):621-633

5 Kim DH, Murovic JA, Tiel RL, Kline DG. Management and outcomes in 318 operative common peroneal nerve lesions at the Louisiana State University Health Sciences Center. Neurosurgery 2004;54 (06):1421-1428

6 Kim DH, Ryu S, Tiel RL, Kline DG. Surgical management and results of 135 tibial nerve lesions at the Louisiana State University Health Sciences Center. Neurosurgery 2003;53(05):1114-1124

7 Kim DH, Murovic JA, Tiel R, Kline DG. Management and outcomes in 353 surgically treated sciatic nerve lesions. J Neurosurg 2004; 101(01):8-17

8 Matejcík V. Peripheral nerve reconstruction by autograft. Injury 2002;33(07):627-631

9 Aydin A, Ozkan T, Aydin HU, et al. The results of surgical repair of sciatic nerve injuries. Acta Orthop Traumatol Turc 2010;44(01): 48-53

10 Andersen HL, Andersen SL, Tranum-Jensen J. Injection inside the paraneural sheath of the sciatic nerve: direct comparison among ultrasound imaging, macroscopic anatomy, and histologic analysis. Reg Anesth Pain Med 2012;37(04):410-414
11 Roganović Z, Pavlićević G, Petković S. Missile-induced complete lesions of the tibial nerve and tibial division of the sciatic nerve: results of 119 repairs. J Neurosurg 2005;103(04):622-629

12 Gousheh J, Babaei A. A new surgical technique for the treatment of high common peroneal nerve palsy. Plast Reconstr Surg 2002;109 (03):994-998

13 Samardzić MM, Rasulić LG, Vucković CD. Missile injuries of the sciatic nerve. Injury 1999;30(01):15-20

14 Wood MB. Peroneal nerve repair. Surgical results. Clin Orthop Relat Res 1991;(267):206-210

15 Gousheh J, Arasteh E, Beikpour H. Therapeutic results of sciatic nerve repair in Iran-Iraq war casualties. Plast Reconstr Surg 2008; 121(03):878-886

16 Kalomiri DE, Soucacos PN, Beris AE. Nerve grafting in peripheral nerve microsurgery of the upper extremity. Microsurgery 1994; 15(07):506-511

17 Terzis JK, Kokkalis ZT. Selective contralateral c7 transfer in posttraumatic brachial plexus injuries: a report of 56 cases. Plast Reconstr Surg 2009;123(03):927-938

18 Kline DG. Nerve surgery as it is now and as it may be. Neurosurgery 2000;46(06):1285-1293

19 Kim DH, Kline DG. Management and results of peroneal nerve lesions. Neurosurgery 1996;39(02):312-319

20 Lee YH, Chung MS, Gong HS, Chung JY, Park JH, Baek GH. Sural nerve autografts for high radial nerve injury with nine centimeter or greater defects. J Hand Surg Am 2008;33(01):83-86 